\title{
Production of Bacteriocin Like Compound from Lactic Acid Bacteria and Screening its Therapeutic Potential
}

\section{Rashmi Gaikwad, Vinal Pardhi* and Valencia D'Souza}

National Facility for Biopharmaceuticals, G N Khalsa College, Matunga, Mumbai, India

*Corresponding Author: Vinal Pardhi, National Facility for Biopharmaceuticals, G N

Khalsa College, Matunga, Mumbai, India.
Received: August 19, 2020

Published: October 22, 2020

(C) All rights are reserved by Vinal Pardhi., et al.

\section{Abstract}

Antimicrobial peptides (AMPs) have emerged to be promising alternatives to traditional antibiotics due to their broad spectrum activity. Bacteriocins are AMPs that have shown immense potential for diversified use in the food and pharma industry, agriculture and apiculture. Strains of Lactic Acid Bacteria (LAB) produce a wide variety of AMPs. They are considered safe additives in food which makes them a potential candidate to replace abusive use of therapeutic antibiotics which leads to bacterial antibiotic resistance. In the current work, bacteriocin like compounds (BLC) were isolated from Lactobacillus fermentum and evaluated for their anti-microbial and anti-cancer properties. They were further concentrated by employing acetone precipitation and analysed using Tricine SDS-PAGE technique to determine the peptide nature and molecular weight which was found to be approx. $5 \mathrm{kDa}$ after in-situ antibacterial activity assay. Antimicrobial activity of these peptides was investigated using agar cup diffusion method and showed significant bactericidal activity against B. subtilis, S. aureus, K. pneumonia and S. epidermidis. The anti-cancer studies of the partially purified peptides displayed notable activity against MCF 7 breast cancer cell lines. The bacteriocin like compound isolated from Lactobacillus fermentum exhibits the potential of being a challenging molecule to replace traditional antibiotics if a reproducible, scalable and cost effective process is developed.

Keywords: Antimicrobial Peptides; Bacteriocin; Lactobacillus fermentum; Partial Purification; Tricine-PAGE

\section{Abbreviations}

AMPs: Antimicrobial Peptides; LAB: Lactic Acid Bacteria; L. fermentum: Lactobacillus fermentum; BLC: Bacteriocin Like Compounds; B. subtilis: Bacillus subtilis; S. aureus: Staphylococcus aureus; K. pneumonia: Klebsiella pneumonia; S. epidermidis: Staphylococcus epidermidis; SDS-PAGE: Sodium Dodecyl Sulfate-Polyacrylamide Gel Electrophoresis; DMEM: Dulbecco's Modified Eagle Medium; RPMI: Roswell Park Memorial Institute; MTT: 3-(4,5-dimethylthiazol-2-yl)-2,5-diphenyl Tetrazolium Bromide; kDa: Kilodalton

\section{Introduction}

Despite of advancement in medical sector to overcome pathogenic infections in humans, emergence and dissemination of re- sistant bacterial species has been a global threat. Nosocomial infections caused by multidrug resistant pathogens has remained a prominent issue in healthcare field [1]. The current antibiotics are failing to combat the antimicrobial resistance peril. Bacteriocins offer a promising potential as a substitute, for combating MDR pathogenic infections that have proven a threat to public health. The discovery of such antimicrobial peptides/proteins (AMPs) exhibited a broad spectrum of inhibition activity against these MDR organisms $[1,2]$. Antimicrobial peptides or proteins produced by bacteria are categorized as bacteriocins having low molecular weight [3]. The first description of bacteriocin-mediated inhibition was reported 80 years ago, when antagonism between strains of Escherichia coli was first discovered [4]. Many substances produced by animals, 
plants, insects, and bacteria possess antimicrobial activities. The production is triggered due to scant nutrients in the production environment giving rise to a variety of bacteriocins [5]. More than $99 \%$ of bacteria can produce at least one bacteriocin, most of which are not identified. The genes for bacteriocins encodes ribosomally synthesized AMPs that show bactericidal activity against related/ non-related microbes as one of the inherent defence mechanism of bacteria [4].

Some bacteriocins show desirable properties for in vivo application, such as stability to low $\mathrm{pH}$ and heat, simple production and extraction processes and little, if any, inhibitory activity towards eukaryotic cells. Therefore, bacteriocins have been evaluated as the most promising class of antimicrobial peptides to be used as antibiotic substitutes in the field of animal and human medicine or in designing and production of new antimicrobials [6]. Various systemic urogenital, gastrointestinal, respiratory and skin infections, including infections caused by multidrug-resistant bacteria can be well tackled by bacteriocins as per early reports [2].

An alternative for the traditional antibiotic system was discovered by the studies on bacteriocins and its derivatives. Bacteriocins can be termed as next generation antibiotics. Many of the ongoing research shows its role in oncological treatments. In order to understand the in-depth mechanism of these new generation antibiotics, researchers can study the combinatorial or individual effect of bacteriocin or its coding gene with other multiple genes. The upregulation or downregulation of cancer biomarkers known to the world; due to bacteriocin action, can lead to a breakthrough in cancer research [7]. Bacteriocins are highly heterogeneous peptides in terms of size, structure and activity and are very potent and effective at pico- or nanomolar concentrations [8]. It initiates the cell death process by forming a channel in the target cell membrane causing passage of low-molecular-weight ions out of cells, leading to collapse of proton motive force [2].

Past reports emphasize on the ability of lactic acid bacteria (LAB) to naturally produce bacteriocins. Rea., et al. (2010) have shown that bacteriocin-producing probiotics play an important role in the fight against infectious bacteria in the human gastrointestinal tract. LABs are classified under "generally recognized as safe" (GRAS), thus the bacteriocins produced by the LAB are also accepted as safe. Nisin and Pediocin that are commercially available bacteriocins show superior inhibitory activity against various pathogenic or saprophytic bacteria in food, either alone or in synergy with other drugs $[9,10]$. Many bacteriocins are produced by food-grade lactic acid bacteria (LAB), and this offers the possibility of manipulating food microbial ecosystems in a deliberate fashion for example, by using bacteriocins to protect food against contamination, or prevent the growth of specific pathogenic bacteria [4]. To explore the plethora of activities such as anticancer, antiviral, antifungal, spermicidal etc. exhibited by bacteriocins, researchers have taken interest in production of these molecules by LAB from various sources [11,12]. An attempt was made for the production, purification and characterization of bacteriocin from marine bacterium Lactobacillus murinus AU06 (L. murinus). In addition to this, purified bacteriocin was tested for its antimicrobial activity against fish pathogens [13]. At present, they are mainly obtained for structural research since the yield of the purified product was accounted for a few percent. The usual procedure involves the bacteriocin precipitation of the culture liquid by ammonium sulfate, hydrophobic chromatography on octyl Sepharose, ion exchange chromatography and high-performance liquid chromatography. Probably, the low yield of the purified product is one of the reasons for the lack of examples of medical use of bacteriocins. $\mathrm{OH}$., et al. conducted a study which stated that a $3.5 \mathrm{kDa}$ bacteriocin was obtained after hydrophobic interaction column chromatography using Octyl-Sepharose CL-4B (Pharmacia Biotec AB, Uppsala, Sweden) [14]. The current work describes the isolation and characterization of a bacteriocin-like compound produced by Lactobacillus fermentum that shows an antimicrobial activity against potential human pathogens and shows evident anti- cancer activity on MCF-7 cell lines.

\section{Materials and Methods}

Reagents and chemicals

Acetone (Molychem), Ethanol (Molychem), Tris buffer (Merck), DMEM (Thermo scientific - 11995065), Primocin (Biogene India - ant-pm-2), FBS (Thermo Fisher Scientific - 10437028), PBS (Thermo Fisher Scientific -10010023), 0.5\% Trypsin EDTA (Thermo Fisher Scientific -15400054), Lactobacillus MRS broth (HiMedia), Trypan Blue (HiMedia -TC193), DMSO (Sigma -D8418), MTT - HiMedia (TC191) were procured. MCF-7 cell lines were procured from National center for cell sciences (NCCS), Pune.

All electrophoresis reagents were procured from Amresco, except tricine which was of sigma make. Vertical electrophoretic system from BioRad was used with BioRad gel caster assembly.

\section{Strains and culture conditions}

The strain for carrying out the present study was procured from National Facility for Biopharmaceuticals (NFB), G.N. Khalsa College. The strain Lactobacillus fermentum VDLB03 strain was iso- 
lated from chicken gut and showed percent identity of 91\% with reference sequences having NCBI accession no of MH100902. Bacillus subtilis (MTCC 1789), Klebsiella pneumoniae (ATCC 10031), Staphylococcus aureus (MTCC 96) and Staphylococcus epidermidis (MTCC435). were used as test organisms in the study.

BLC production using shake flask

Lactobacillus MRS broth as a seed medium was prepared as per the manufacturer's instructions. For bacteriocin production, growth of the organism was carried out in 1 litre Erlenmeyer flasks containing production medium along $10 \%$ oil as an inducer. Approximately, 1.0 OD inoculum was added into the media and the flasks were incubated at $37^{\circ} \mathrm{C}$ in an incubator shaker at $180 \mathrm{rpm}$ for $96 \mathrm{~h}$. After the desired incubation period, biomass was harvested and the culture filtrate was analyzed for bacteriocin production. The fermented broth was centrifuged at 10,000 rpm/33000g for 10 mins to separate the bacterial cells so as to obtain the clear supernatant.

\section{Isolation of BLC}

Isolation of peptides was performed using the acetone precipitation. Chilled acetone was added to crude extract (concentrated) in the ratio of $4: 1(\mathrm{v} / \mathrm{v})$ in acetone compatible falcon tubes. The tubes were vortexed and placed at $-20^{\circ} \mathrm{C}$ for protein precipitation for $2 \mathrm{~h}$. The precipitate was then centrifuged at $10000 \mathrm{rpm}, 4^{\circ} \mathrm{C}$ for 15 minutes. Post centrifugation, the supernatant collected was kept at $60^{\circ} \mathrm{C}$ till the complete evaporation of acetone. The pellet obtained was weighed and reconstituted in $10 \mathrm{mM}$ Tris pH 8.0 at a concentration of $10 \mathrm{mg} \mathrm{mL}^{-1}$ and stored at $4^{\circ} \mathrm{C}$ till further use.

Determination of the molecular weight of BLC by tricine SDSPAGE

After precipitation, the samples were loaded on $16 \%$ tricine PAGE to determine the presence of peptides. Silver staining was performed for detection of low molecular weight proteins or peptides.

\section{Antimicrobial activity}

A well diffusion method was used to check the activity of crude BLC in from the strain. Four pathogenic bacterial strains were selected for assessing the antimicrobial activity of the sample. An overnight culture of the indicator strains was used to inoculate appropriate agar growth media at $37^{\circ} \mathrm{C}$. The culture OD was adjusted to McFarland standard 0.5 in order to get $1.5 \times 10^{8} \mathrm{CFU} / \mathrm{ml}$. The bacterial cell suspension was mixed to homogeneity to give a final density of $1 \times 10^{6} \mathrm{CFU} / \mathrm{ml}$. Wells of $6 \mathrm{~mm}$ diameter were punctured aseptically into the agar using a cork borer. $0.1 \mathrm{~mL}$ of filtered cellfree supernatant obtained from L. fermentum grown in MRS broth was added to each well along with the controls and allowed to diffuse into the agar for $2 \mathrm{~h}$ at room temperature. Subsequently, the plates were incubated at $37^{\circ} \mathrm{C}$ and examined after $24 \mathrm{~h}$ for inhibition zones in millimeter $(\mathrm{mm})$.

\section{Cytotoxicity study}

Cell lines and growth conditions

Cancer cell line (Human) MCF-7 (breast cancer) was utilized to study the probable anticancer effects of the isolated compounds (bacteriocins) on tumor cells. The cells were routinely grown in RPMI medium (Gibco) supplemented with 10\% heat-inactivated (30 min, $56^{\circ} \mathrm{C}$ ) FBS (Gibco) and 1\% primocin mixture. All experiments were performed at $37^{\circ} \mathrm{C}$ in $5 \% \mathrm{CO}_{2}$.

\section{MTT assay}

The cytotoxicity of BLC MCF-7 cell line was determined by MTT colorimetric assay. The cell lines used in this study were obtained from the National Center for Cell Sciences. Cells $\left(1 \times 10^{4}\right.$ cells/ well) in DMEM containing 10\% fetal bovine serum were seeded into 96-well flat bottom culture plates (Nunc A/S, Denmark). After $24 \mathrm{~h}$ of incubation, at $37^{\circ} \mathrm{C}$ in $5 \% \mathrm{CO}_{2}$ humidified atmosphere, various concentrations of each fractions (final concentrations from $10 \%$ to $100 \%$ ) were added to the culture and incubated for $24 \mathrm{~h}$ at $37^{\circ} \mathrm{C}$ in $5 \%$ $\mathrm{CO}_{2}$ humidified atmosphere. Then cells were added along with $10 \mu \mathrm{L} /$ well of MTT $(5 \mathrm{mg} / \mathrm{mL})$ and incubated for $4 \mathrm{~h}$ in incubator at $37^{\circ} \mathrm{C}$ in $5 \% \mathrm{CO}_{2}$ humidified atmosphere. The reaction was stopped by 100 $\mu \mathrm{L}$ of DMSO. The plate was then incubated and agitated for $10 \mathrm{~min}$. The absorbance of each well was read at $570 \mathrm{~nm}$ wavelength in ELISA Reader (Biorad, USA), using wells without cells as blanks. All experiments were performed in triplicate. The effect of all fractions on the proliferation of human breast cancer cells was expressed as:

$\%$ cyto-viability, using the following formula: \% Cyto-viability = (Absorbance of treated cells/Absorbance of control cells) x $100 \%$.

\section{Results and Discussion}

$L$ fermentum is a LAB, usually isolated from milk and food sources. These bacteria fall under the group of GRAS organisms, thus are safe to use for the production of bacteriocins which in-turn are widely used as food preservatives. This strain of L. fermentum VDLB03 was previously isolated, identified with classical biochemical methods as well as using molecular identification tool such as 16 rRNA sequencing. 
Bacteriocins predominantly exhibit antibacterial activity, thus at each step of the cultivation process the antibacterial activity was assessed against $B$. subtilis which is a nonpathogenic bacterium, $\mathrm{S}$. aureus a common pathogen, K. pneumoniae an opportunistic pathogen and S. epidermidis a biofilm producer bacterium.
Figure 1 represent the antimicrobial activity of bacteriocin produced by L. fermentum against B. subtilis, S. aureus, K. pneumoniae and $S$. epidermidis respectively. The positive control is Chloramphenicol and the negative control is DMSO. Sample A, B, C and D represents 24, 48, 72 and 96 hours of culture supernatant of $L$. fermentum.

\begin{tabular}{|l|c|c|c|c|c|c|c|c|}
\hline \multicolumn{7}{|c|}{ Inhibition zone diameter in mm } \\
\hline \multirow{2}{*}{ Sample ID } & B. subtilis & \multicolumn{2}{|c}{ S. aureus } & \multicolumn{2}{c|}{ K. pneumonia } & \multicolumn{2}{c|}{ S. epidermidis } \\
\hline & MRS & MNM & MRS & MNM & MRS & MNM & MRS & MNM \\
\hline \multirow{2}{*}{+ ve ctl } & 18 & 26 & 22 & & 23 & 26 & 19 & 24 \\
\cline { 2 - 10 } & ++ & +++ & +++ & & +++ & +++ & ++ & +++ \\
\hline \multirow{2}{*}{-ve ctl } & 0 & 0 & 0 & & 0 & 0 & 0 & 0 \\
\cline { 2 - 10 } & - & - & - & & - & - & - & - \\
\hline \multirow{2}{*}{ A } & 12 & 12 & 10 & & 10 & 8 & 10 & 9 \\
\cline { 2 - 10 } & + & & + & & + & + & + & + \\
\hline \multirow{2}{*}{ B } & 12 & 13 & 9 & & 9 & 8 & 11 & 10 \\
\cline { 2 - 10 } & + & & + & & + & + & + & + \\
\hline \multirow{2}{*}{ C } & 13 & 14 & 10 & & 11 & 8 & 12 & 11 \\
\cline { 2 - 10 } & + & & + & & + & + & + & + \\
\hline \multirow{2}{*}{ D } & 13 & 14 & 12 & & 12 & 8 & 12 & 12 \\
\cline { 2 - 9 } & + & & + & & + & + & + & + \\
\hline
\end{tabular}

Table: Antimicrobial spectrum study exhibited by BLC.

'+++': High inhibition, '++': Moderate inhibition, '+': Low inhibition '-': No inhibition.
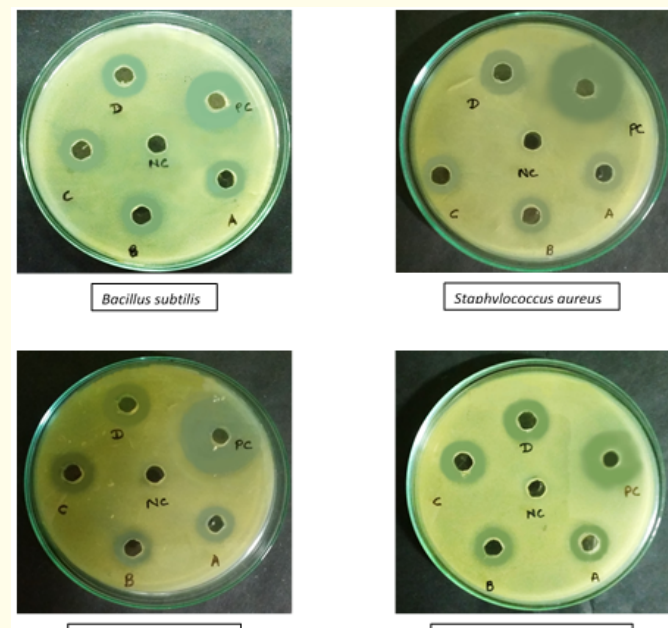

Klebsiella pneumoniae

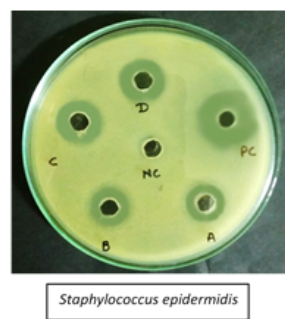

Figure 1: Antimicrobial activity determination of crude BLC prepared from L. fermentum cultivated up to $96 \mathrm{~h}$ by agar cup diffusion method. Each well was seeded with $100 \mu \mathrm{L}$ of crude extract, chloramphenicol was used as positive control and blank sterile media was used as negative control. Plates were observed for zones after 24 hours and captured.
Zone of inhibition was observed for all the samples loaded in the well against all the test organisms. Although, the cultivation time differs from 24 hours to 96 hours, when the activity of culture supernatant was assessed, in 96 hours culture supernatant, slightly improved activity exhibited by bacteriocins was observed. The lactic acid bacteria used in the current study was isolated from chicken gut. Bacteriocins from various strains of Lactic acid bacteria from variety of sources have been reported earlier. In one of the recent studies bacteriocins from Enterococcus faecalis KT11 isolated from traditional Kargı Tulum cheese has been reported to show antagonistic effect against various gram positive as well as gram negative bacteria [9].

Electrophoretic technique reveals the peptide nature of the compound, and Tricine PAGE has been reported for resolution of small molecular weight proteins, peptides and also specifically for bacteriocin analysis [16,17]. Bacterocin isolated from lactic acid bacteria such as Nisin, lactocin, pediocin have low molecular weight and are mostly $<10 \mathrm{kDa}$, on the contrary some have high molecular weight upto $30 \mathrm{kDa}$ as well [7] [18]. As can be seen in figure 2, the molecular weight of BLC was found to be of a low molecular weight 
of approx. $5 \mathrm{kDa}$. Bacteriocins isolated from $L$ fermentum have been reported to be of low molecular weight fermenticin in class II bacteriocine [19] correlating with the data inferred from figure 2 that the obtained peptides could be of same characteristics.

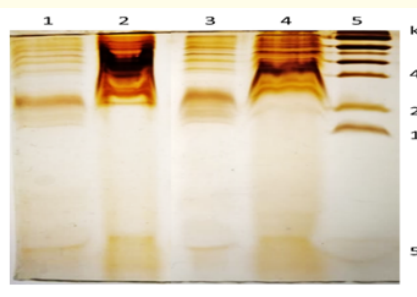

a)

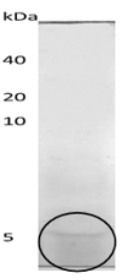

b)

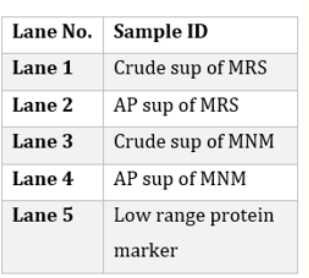

b)
Figure 2: Analysis of BLC and its molecular weight information. The tricine SDS-PAGE of BLC and detection on gel. a) 16\% Tricine

PAGE, silver stained showing BLC from culture supernatant before and after acetone precipitation. b) The half part of the gel was subjected to direct in-situ antibacterial activity assay by the zone of inhibition around the band.

Cytotoxicity assay for determination anticancer potential of BLC

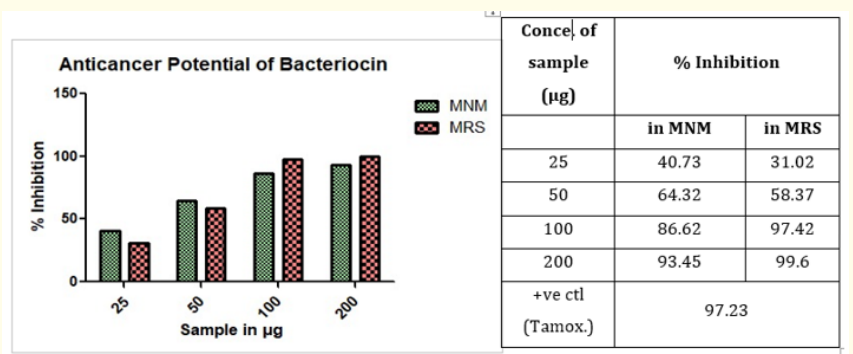

Figure 3: Comparative analysis of in-vitro cytotoxicity activity of BLC produced in two different media against MCF-7.

The $\mathrm{IC}_{50}$ values of MRS and MNM are found to be 34.72 and 44.24 $\mu$ g respectively for BLC obtained from different seed medi$\mathrm{um}$. To calculate $\mathrm{IC}_{50}$ value the best fit graph was chosen in order to avoid deviation. As figure 3 shows the primitive data for anticancer potential, the experiments performed were not in replicates. Further studies would establish closer insights for its anticancer activity. The current results show correlation with the previous reports where such compounds with antibacterial activities have also been screened for its other applications such as anticancer, antiviral and antifungals.
Considering the cost of the anticancer molecules, bacteriocins are easy and cost-effective lead molecules to test against cancer cells. BLC obtained from L. fermentum exhibits considerable anticancer action against MCF-7 cells, although the mechanism involved is still not elucidated. Many of the studies report bacteriocin induces the apoptotic pathway or depolarization of the cell membrane which leads to permeability changes.

\section{Conclusion}

In conclusion, in this paper we have demonstrated that the antimicrobial activity of L. fermentum is mediated by the bacteriocin like compound. In addition to exhibiting an antimicrobial activity against both representatives Gram-positive and Gram-negative bacteria, this compound can also cause the reduction of cancer cells viability. Considering proven antibacterial actions, bacteriocin can be an answer to global antimicrobial resistant threat. The demand for novel entities as a drug candidate to combat pathogenesis due to resistant strains could be fulfilled by the effective bacteriocins. It can be referred as next generation antibiotics. Further, the potential of these bacteriocin like compounds to serve as synergistic agents to conventional antibiotics and cancer drugs should also be tested in order to design safer and potential alternative to current treatment/therapy.

\section{Bibliography}

1. E Meade., et al. "Bacteriocins, potent antimicrobial peptides and the fight against multi drug resistant species: Resistance is futile?". Antibiotics 9.1 (2020).

2. LM T Dicks., et al. "A review: The fate of bacteriocins in the human gastro-intestinal tract: Do they cross the gut-blood barrier?". Frontiers in Microbiology 9 (2018): 1-14.

3. V Juturu and JC Wu. "Microbial production of bacteriocins: Latest research development and applications". Biotechnology Advances 36.8 (2018): 2187-2200.

4. PD Cotter., et al. "Bacteriocins: developing innate immunity for food”. Nature Reviews Microbiology 3.10 (2005): 777-788.

5. SC Yang., et al. "Antibacterial activities of bacteriocins: Application in foods and pharmaceuticals". Frontiers in Microbiology 5 (2014): 1-10.

6. LD Bemena., et al. "Applications of bacteriocins in food, livestock health and medicine.". International Journal of Current Microbiology and Applied Sciences 3.12 (2014): 924-949.

7. VPASP Rashmi Gaikwad. "Bacteriocins and its applications: an overview". Asian Journal of Microbiology Biotechnology 3.3 (2018): 96-104. 
8. M Hassan., et al. "Natural antimicrobial peptides from bacteria: Characteristics and potential applications to fight against antibiotic resistance". Journal of Applied Microbiology 113.4 (2012): 723-736.

9. HS Abanoz and B Kunduhoglu. "Antimicrobial activity of a bacteriocin produced by enterococcus faecalis kt11 against some pathogens and antibiotic-resistant Bacteria". Korean Journal for Food Science of Animal Resources 38.5 (2018): 1064-1079.

10. MP Zacharof., et al. "Bacteriocins: safe, natural antimicrobials for food preservation". International Journal of Food Microbiology 2.1 (2011): 90.

11. L Silkin., et al. "Spermicidal bacteriocins: Lacticin 3147 and subtilosin A". Bioorganic and Medicinal Chemistry Letters 18.10 (2008): 3103-3106.

12. SS Grosu-Tudor., et al. "Characterization of some bacteriocins produced by lactic acid bacteria isolated from fermented foods". World Journal of Microbiology and Biotechnology 30.9 (2014): 2459-2469.

13. S Elayaraja., et al. "Production, purification and characterization of bacteriocin from Lactobacillus murinus AU06 and its broad antibacterial spectrum". Asian Pacific Journal of Tropical Biomedicine 4.1 (2014): S305-S311.

14. S Oh., et al. "Characterization and purification of a bacteriocin produced by a potential probiotic culture, Lactobacillus acidophilus 30SC". Journal of Dairy Science 83.12 (2000): 27472752.

15. H Schägger. "Tricine-SDS-PAGE”. Nature Protocol 1.1 (2006): 16-22.

16. T Huang., et al. "Purification and Characterization of a Novel Cold Shock Protein-Like Bacteriocin Synthesized by Bacillus thuringiensis". Scientific Report 6 (2016): 1-15.

17. J Zhang., et al. "Purification and partial characterization of bacteriocin Lac-B23, a novel bacteriocin production by lactobacillus plantarumJ23, isolated from Chinese traditional fermented milk". Frontiers in Microbiology 9 (2018): 1-7.

18. MP Mokoena. "Lactic acid bacteria and their bacteriocins: Classification, biosynthesis and applications against uropathogens: A mini-review". Molecules 22.8 (2017).

19. B Kaur., et al. "Biomedical applications of fermenticin HV6b isolated from lactobacillus fermentum HV6b MTCC10770". BioMed Research International (2013).

\section{Assets from publication with us}

- Prompt Acknowledgement after receiving the article

- Thorough Double blinded peer review

- Rapid Publication

- Issue of Publication Certificate

- High visibility of your Published work

Website: https://www.actascientific.com/

Submit Article: https://www.actascientific.com/submission.php

Email us: editor@actascientific.com

Contact us: +919182824667 